23

\title{
Повышение устойчивости спектральных характеристик интерференционных покрытий к отклонению в параметрах слоев, входящих в их состав
}

\author{
() Нго Тхай Фи, Л.А. Губанова, Фам Ван Хоа \\ Университет ИТМО, \\ 197101 Санкт Петербург, Россия \\ e-mail: ngothaiphibn@gmail.com, la7777@mail.ru
}

Поступила в редакцию 13.02.2018 г.

\begin{abstract}
Представлен новый метод повышения устойчивости спектральных характеристик интерференционных покрытий к отклонениям в параметрах слоев, входящих в их состав. С помощью этого метода возможно получить спектральную характеристику энергетических коэффициентов пропускания (или отражения) экспериментального изготовленного покрытия, которая максимально приближена к характеристике синтезированного. Представлен способ использования нескольких образцов для контроля толщины слоев в процессе их формирования с целью уменьшения отклонения оптических слоев в процессе изготовления интерференционных покрытий.
\end{abstract}

DOI: $10.21883 /$ OS.2018.08.46374.43-18

Для изменения физико-химических свойств оптических элементов на их преломляющих и отражающих поверхностях формируются интерференционные покрытия, состоящие из нескольких слоев, толщина которых определяет спектральную характеристику отраженного и прошедшего световых потоков. Точность изготовления этих слоев позволяет получать покрытия с требуемыми спектральными характеристиками.

В состав современных интерференционных покрытий входят десятки слоев, полученных при синтезе покрытий, их оптические толщины имеют значения, равные дробным частям некоторой длины волны [1-6]. Реальные величины оптических толщин слоев, входящих в состав интерференционного покрытия, определяются точностью контроля в процессе их изготовления.

Рассмотрим метод увеличения устойчивости спектральных характеристик интерференционных покрытий к отклонениям в параметрах слоев. Обозначим реальную спектральную характеристику энергетического коэффициента пропускания покрытия, состоящего из $k$ слоев, функцией $T\left(n_{i} d_{i}, i, \lambda\right)$. Спектральную характеристику энергетического коэффициента пропускания покрытия, изменяющуюся по мере увеличения оптической толщины $i$-го слоя $(1<i<k)$ в процессе сформирования, $T\left(n_{i}^{\prime} d_{i}^{\prime}, i, \lambda\right)$, где $n_{i} d_{i}$ - оптическая толщина $i$-го слоя, полученная в результате синтеза, $n_{i}^{\prime} d_{i}^{\prime}=n_{i} d_{i}+\Delta n_{i} d_{i}$ - толщина реально изготовленного слоя. Оптические толщины слоев в процессе их формирования часто контролируются по достижению экстремума пропускания [2] на некоторой длине волны. Относительное значение погрешности в регистрации экстремума пропускного сигнала, которое вносит отклонения в оптическую толщину $i$-го слоя, на контрольной длине волны можно определить с помощью разложения в ряд Тейлора

$$
\begin{aligned}
T\left(n_{i}^{\prime} d_{i}^{\prime}, i, \lambda_{0}\right)= & T\left(n_{i} d_{i}, i, \lambda_{0}\right)+\frac{\partial T\left(n_{i} d_{i}, i, \lambda_{0}\right)}{\partial n_{i} d_{i}} \Delta n_{i} d_{i} \\
& +\frac{\partial^{2} T\left(n_{i} d_{i}, i, \lambda_{0}\right)}{\partial n_{i} d_{i}^{2}}\left(\Delta n_{i} d_{i}\right)^{2}+\ldots,
\end{aligned}
$$

тогда

$$
\begin{aligned}
\Delta T\left(i, \lambda_{0}\right)= & \frac{\partial T\left(n_{i} d_{i}, i, \lambda_{0}\right)}{\partial n_{i} d_{i}} \Delta n_{i} d_{i}+\frac{\partial^{2} T\left(n_{i} d_{i}, i, \lambda_{0}\right)}{\partial n_{i} d_{i}^{2}} \\
& \times\left(\Delta n_{i} d_{i}\right)^{2}+\ldots \approx \frac{\partial T\left(n_{i} d_{i}, i, \lambda_{0}\right)}{\partial n_{i} d_{i}} \Delta n_{i} d_{i},
\end{aligned}
$$

где $\lambda_{0}$ - контрольная длина волны.

Отсюда получаем

$$
C\left(i, \lambda_{0}\right)=\left|\frac{\Delta T\left(i, \lambda_{0}\right)}{\Delta n_{i} d_{i}}\right|=\left|\frac{\partial T\left(n_{i} d_{i}, i, \lambda_{0}\right)}{\partial n_{i} d_{i}}\right| .
$$

Очевидно, что чем больше величина $C\left(i, \lambda_{0}\right)$, тем меньше отклонение в оптической толщине изготавливаемого слоя от толщины, полученной в результате синтеза. Поэтому, возможно, следует добавить величину $C$ в процесс синтезирования структуры покрытия. Уменьшение значения величины $C$ в условии синтеза позволит повысить устойчивость интерференционного покрытия.

Для увеличения устойчивости синтезированного покрытия в работе [7] представлен метод повторной оптимизации структуры покрытия после изготовления нескольких слоев с использованием представленной в настоящей работе функции качества, что позволит определить оптические толщины остальных слоев, которые обеспечат спектральные характеристики изготовленного покрытия, максимально приближенные к требуемым. 
Однако в процессе изготовления слоя, ограниченного воздухом, отсутствуют условия для его оптимизации. Если отклонение оптической толщины этого слоя значительно влияет на спектральные характеристики коэффициента пропускания (или отражения) интерференционного покрытия, то полученное экспериментальное покрытие не будет удовлетворять требуемому условию. Поэтому в этой работе рассматривается метод получения спектральных характеристик энергетического коэффициента пропускания (или отражения) изготавливаемых покрытий, характеристики которых близки к характеристикам синтезированного.

В общем виде спектральная характеристика любого интерференционного покрытия $T(\lambda)$ (или $R(\lambda)$ ) должна удовлетворять следующим условиями: $T(\lambda)<T_{1}$ в диапазоне $\lambda_{a}-\lambda_{1}, T(\lambda)>T_{2}$ в диапазоне $\lambda_{1}-\lambda_{2}, T(\lambda)<T_{3}$ в диапазоне $\lambda_{2}-\lambda_{3}, T(\lambda)>T_{4}$ в диапазоне $\lambda_{3}-\lambda_{b}$, где $T_{1}, T_{2}, T_{3}, T_{4}$ - значения энергетического коэффициента отражения, удовлетворяющие техническому заданию, $\lambda_{a}-\lambda_{b}-$ рассматриваемый спектральный диапазон. Для определения оптических толщин каждого слоя будем использовать функцию качества $F(X)$, которая может быть написана в виде

$$
F(X)=\lim _{L \rightarrow \infty} \sum_{j=1}^{L}\left|F\left(X, \lambda_{i}\right)\right|,
$$

где

$$
\begin{aligned}
& F\left(X, \lambda_{j}\right) \\
& \quad= \begin{cases}0 \text { в }\left[\lambda_{a}-\lambda_{1}\right] \text { или }\left[\lambda_{2}-\lambda_{3}\right], & \text { если } \Delta T \leq 0, \\
\Delta T \Delta \lambda \text { в }\left[\lambda_{a}-\lambda_{1}\right] \text { или }\left[\lambda_{2}-\lambda_{3}\right], & \text { если } \Delta T \geq 0, \\
|\Delta T| \Delta \lambda \text { в }\left[\lambda_{1}-\lambda_{2}\right] \text { или }\left[\lambda_{3}-\lambda_{b}\right], & \text { если } \Delta T \leq 0, \\
0 \text { в }\left[\lambda_{1}-\lambda_{2}\right] \text { или }\left[\lambda_{3}-\lambda_{b}\right], & \text { если } \Delta T \geq 0,\end{cases}
\end{aligned}
$$

$\Delta \lambda=\left(\lambda_{a}-\lambda_{b}\right) / L, L-$ количество отрезков спектрального интервала от $\lambda_{a}$ до $\lambda_{b}, X$ - вектор оптических толщин слоев $X=\left\{n_{1} d_{1}, n_{2} d_{2}, \ldots, n_{i} d_{i}, \ldots\right\}$ и $\Delta T=T(X, \lambda)-T(\lambda), T(\lambda)$ - требуемая спектральная характеристика энергетического коэффициента пропускания, $T(X, \lambda)$ - спектральная характеристика энергетического коэффициента пропускания изготовленного покрытия.

Видно, что чем меньше значение функции качества, тем ближе спектральная характеристика коэффициента пропускания к желаемой. Если значение функции качества равно нулю, то спектральные характеристики изготовленного покрытия лучше, чем требовалось.

Если после изготовления первого слоя наблюдается отклонение оптической толщины этого слоя $\Delta n_{1} d_{1}$, то необходимо определить новую оптическую толщину второго слоя, чтобы величина функции качества была минимальной $F\left(X_{1,2}\right)$. При разложении функции, определяющей энергетический коэффициент пропускания в ряд Тейлора, получаем выражение

$$
\begin{aligned}
& T\left(n_{1} d_{1}+\Delta n_{1} d_{1} ; n_{2} d_{2}+\Delta n_{2} d_{2}, \lambda\right) \approx T_{1,2}(\lambda) \\
& +\frac{\partial T_{1,2}}{\partial n_{1} d_{1}} \Delta n_{1} d_{1}+\frac{\partial T_{1,2}}{\partial n_{2} d_{2}} \Delta n_{2} d_{2}+\frac{\partial^{2} T_{1,2}}{\left(\partial n_{2} d_{2}\right)^{2}}\left(\Delta n_{2} d_{2}\right)^{2},
\end{aligned}
$$

тогда

$$
\begin{aligned}
\Delta T_{1,2}(\lambda)= & \frac{\partial T_{1,2}}{\partial n_{1} d_{1}} \Delta n_{1} d_{1}+\frac{\partial T_{1,2}}{\partial n_{2} d_{2}} \Delta n_{2} d_{2} \\
& +\frac{\partial^{2} T_{1,2}}{\left(\partial n_{2} d_{2}\right)^{2}}\left(\Delta n_{2} d_{2}\right)^{2} .
\end{aligned}
$$

Величина функции качества может быть переписана следующим образом:

$$
\begin{aligned}
F_{1,2}= & \Delta \lambda \sum_{j=1}^{L}\left[\frac{\partial T_{1,2}}{\partial n_{1} d_{1}} \Delta n_{1} d_{1}+\frac{\partial T_{1,2}}{\partial n_{2} d_{2}} \Delta n_{2} d_{2}\right. \\
& \left.+\frac{\partial^{2} T_{1,2}}{\left(\partial n_{2} d_{2}\right)^{2}}\left(\Delta n_{2} d_{2}\right)^{2}\right] .
\end{aligned}
$$

Аналогично после изготовления $(i-1)$-го слоя величину функции качества можно определить в виде

$$
\begin{aligned}
F_{1,2, \ldots, i}= & \Delta \lambda \sum_{j=1}^{L} \mid \sum_{h=1}^{i-1} \frac{\partial T_{1,2, \ldots, i}}{\partial n_{j} d_{j}} \Delta n_{j} d_{j}+\frac{\partial T_{1,2, \ldots, i}}{\partial n_{i} d_{i}} \Delta n_{i} d_{i} \\
& +\frac{\partial^{2} T_{1,2, \ldots, i}}{\left(\partial n_{i} d_{i}\right)^{1}}\left(\Delta n_{i} d_{i}\right)^{2} \mid
\end{aligned}
$$

где $\Delta \lambda=\lim _{L \rightarrow \infty}\left(\lambda_{b}-\lambda_{a}\right) / L$.

Обозначим

$$
\begin{gathered}
A=\Delta \lambda \sum_{j=1}^{L} \frac{\partial^{2} T_{1,2, \ldots, i}}{\left(\partial n_{i} d_{i}\right)^{2}} ; \quad B=\Delta \lambda \sum_{j=1}^{L} \frac{\partial T_{1,2, \ldots, i}}{\partial n_{i} d_{i}} ; \\
D=\Delta \lambda\left|\sum_{j=1}^{L} \sum_{h=1}^{i-1} \frac{\partial T_{1,2, \ldots, i}}{\partial n_{j} d_{j}} \Delta n_{j} d_{j}\right| .
\end{gathered}
$$

Тогда уравнение (9) может быть написано в виде

$$
F_{1,2, \ldots, i}=\Delta \lambda\left[A\left(\Delta n_{i} d_{i}\right)^{2}+B \Delta n_{i} d_{i}+D\right] .
$$

Определим минимум функции (10)

$$
\min F_{1,2, \ldots, i}=\Delta \lambda\left[D-\frac{B^{2}}{4 A}\right], \text { когда } \Delta n_{i} d_{i}=-\frac{B}{2 A} .
$$

Предположим, что синтезированная конструкция интерференционного покрытия состоит из $k$-слоев. После изготовления $k$-го слоя всегда наблюдается отклонение оптической толщины этого слоя и не существует рассмотренных выше условий для уменьшения величины функции качества $F(X)$. Если отклонение оптической толщины $k$-го слоя существенно влияет на спектральные 


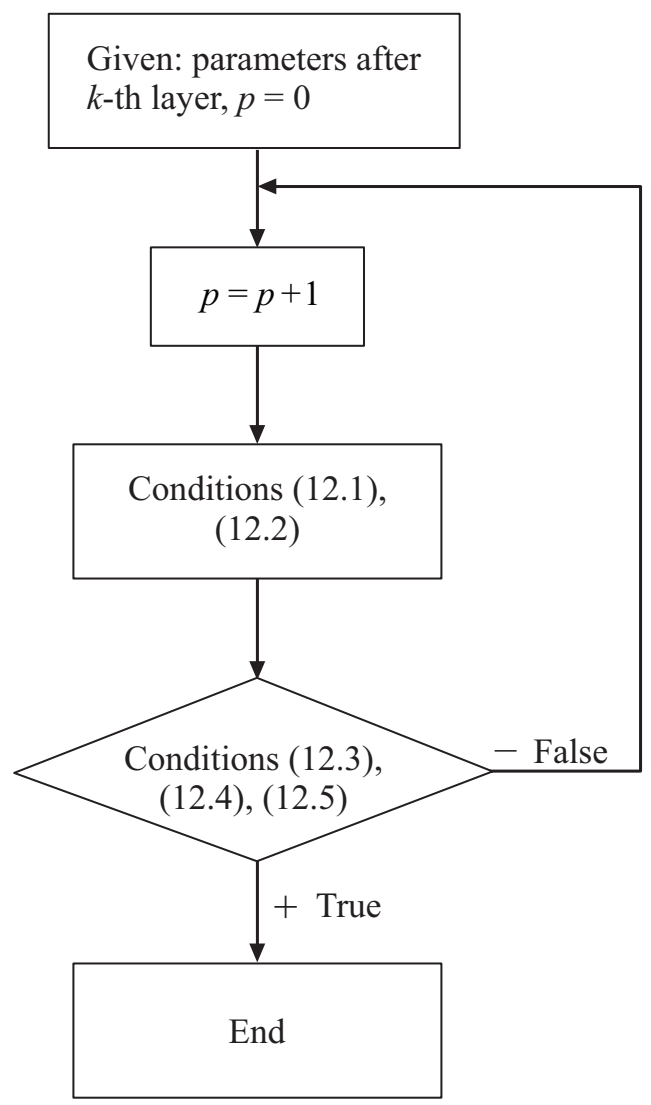

Рис. 1. Схема алгоритма определения дополнительного количества слоев и их параметров.

характеристики рассматриваемого покрытия, то получаемое покрытие не вполне удовлетворяет техническому требованию. В этом случае следует добавить слой (или несколько слоев) в структуру рассматриваемого покрытия для увеличения его устойчивости. Показатели преломления материалов, из которых изготавливаются дополнительные слои, обычно выбирают из условий $1.38 \leq n \leq 2.30$, минимальная оптическая толщина дополнительных слоев составляет 25-30 nm [6]. Поэтому дополнительные слои должны удовлетворять следующим условиям:

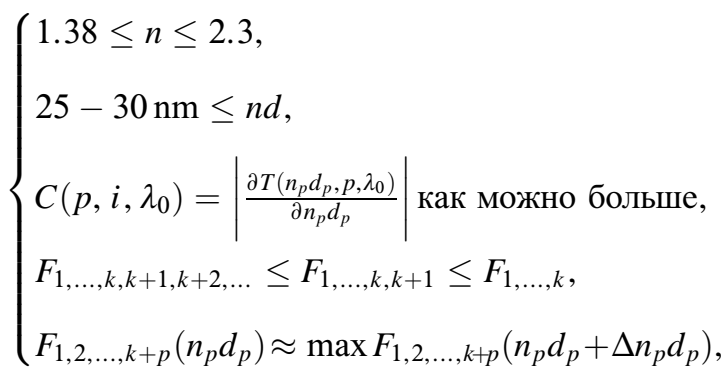

где $p$ - количество дополнительных слоев; $\max \left(F_{1,2, \ldots, k+p}\left(n_{p} d_{p}+\Delta n_{p} d_{p}\right)\right)$ - максимальная величина функции качества при отклонении оптической толщины $-\max \left(\Delta n_{p} d_{p}\right) \leq \Delta n_{p} d_{p}<\max \left(\Delta n_{p} d_{p}\right) ; \max \left(\Delta n_{p} d_{p}\right)$ может быть определено, если погрешность в регистрации экстремума пропускания сигнала известна. Представленное условие (12.3) для уменьшения отклонения оптической толщины слоя, условие (12.4) для получения улучшения спектральной характеристики интерференционного покрытия и условие (12.5) необходимы для уменьшения влияния отклонения оптической толщины $p$-го слоя на спектральную характеристику. Максимальное отклонение оптической толщины $p$-го слоя можно определить из условий (12.3). На рис. 1 представлена схема алгоритма определения количества дополнительных слоев и показателей преломления материалов, из которых они изготавливаются.

Если в природе не существуют материалы, из которых могут изготавливаться дополнительные слои, то эти слои можно заменить симметричной трехслойной системой слоев, толщины и материалы которых выбраны из условия, представленного ниже. Общая схема замены слоя $p$, имеющего фазовую толщину $\Phi_{p}$ и показатель преломления $N_{p}$, представлена на рис. 2 .

Эквивалентный показатель преломления $N_{p}$ и эквивалентная фазовая толщина $\Phi_{p}$ могут быть определены следующим образом [8]:

$$
\begin{aligned}
& N_{p}^{2}=\left(n_{1}^{(3)}\right)^{2} \\
& \times\left[\frac{\sin 2 \varphi_{1}^{(3)} \cos \varphi_{2}^{(3)}+G \cos 2 \varphi_{1}^{(3)} \sin \varphi_{2}^{(3)}-Q \sin \varphi_{2}^{(3)}}{\sin 2 \varphi_{1}^{(3)} \cos \varphi_{2}^{(3)}+G \cos 2 \varphi_{1}^{(3)} \sin \varphi_{2}^{(3)}+Q \sin \varphi_{2}^{(3)}}\right], \\
& \cos \Phi_{p}=\cos 2 \varphi_{1}^{(3)} \cos \varphi_{2}^{(3)}-\frac{1}{2} G \sin 2 \varphi_{1}^{(3)} \sin \varphi_{2}^{(3)},
\end{aligned}
$$

где

$$
\begin{gathered}
G=\frac{1}{2}\left(\frac{n_{1}^{(3)}}{n_{2}^{(3)}}+\frac{n_{2}^{(3)}}{n_{1}^{(3)}}\right) ; Q=\frac{1}{2}\left(\frac{n_{1}^{(3)}}{n_{2}^{(3)}}-\frac{n_{2}^{(3)}}{n_{1}^{(3)}}\right) ; \\
\varphi_{1}^{(3)}=\frac{2 \pi}{\lambda} n_{1}^{(3)} d_{1}^{(3)} ; \quad \varphi_{2}^{(3)}=\frac{2 \pi}{\lambda} n_{2}^{(3)} d_{2}^{(3)} .
\end{gathered}
$$

Из выражений (13) и (14) при условии, что величины $n_{1}^{(3)}, n_{2}^{(3)}$ выбираются из значений коэффициентов преломления реальных пленкообразующих материалов, $n_{2} d_{2}=2 n_{1} d_{1}$ для известных значений $N_{p}, \Phi_{p}$, можно определить величины оптических толщин слоев $\left(n_{1}^{(3)} d_{1}^{(3)}, n_{2}^{(3)} d_{2}^{(3)}\right)$.

Предполагаемые отклонения в толщинах слоев $\partial T\left(n_{p} d_{p}, p, \lambda_{0}\right)$ определяются способом их контроля в

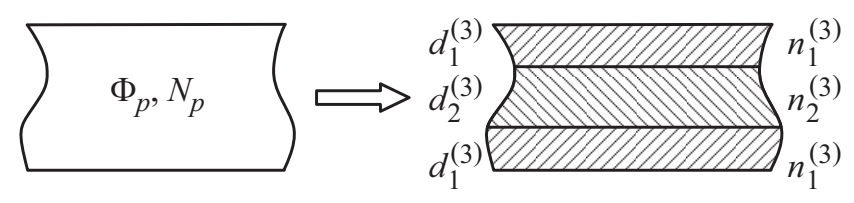

Рис. 2. Схема замены однородного слоя на трехслойную систему: $d_{1}^{(3)}, d_{2}^{(3)}-$ физическая толщина слоев, $n_{1}^{(3)}, n_{2}^{(3)}-$ показатели преломления материалов. 


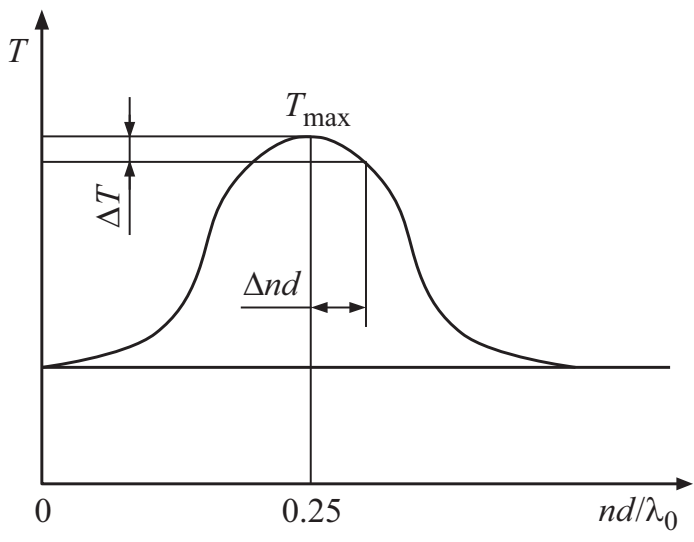

Рис. 3. Измерение энергетического коэффициента пропускания на контрольной длине волны $\lambda_{0}$ при нанесении непоглощающего слоя на оптический элемент, изготовленный из непоглощающего материала.

процессе формирования. В процессе изготовления интерференционных покрытий методом термического испарения в вакууме наиболее часто используется фотометрический метод контроля оптической толщины слоя. Например, вакуумные установки ВУ-1А и ВУ-2М комплектуются системой фотометрического контроля толщины слоев СФКТ-751В. Контроль оптической толщины выполняют по изменению энергетических коэффициентов отражения или пропускания образцов. Контроль фотометрическим методом обычно заключается в том, что испарение пленкообразующего материала прекращается, когда наблюдаемое изменение пропускания (отражения) достигает экстремума или заданной величины [2-4]. В начале формирования слоя наблюдаемая величина пропускания быстро изменяется, а вблизи экстремума сигнал меняется медленно, что не всегда позволяет точно определить толщину слоя. Величину относительного соотношения между погрешностью при измерении экстремального значения коэффициента пропускания $(\Delta T)$ и отклонениям в оптической толщине контролируемого слоя $(\Delta n d)$ можно определить следующим образом:

$$
C=\left|\frac{\Delta T}{\Delta n d}\right|,
$$

где $\Delta T-$ погрешность в регистрации экстремума пропускания сигнала, $\Delta n d-$ отклонение в оптической толщине экспериментально изготовленного покрытия от синтезированного (рис. 3).

В работе $[2,7]$ показано, что погрешность в регистрации экстремума пропускания сигнала фотометрическим методом меньше $0.3 \%\left(\Delta T_{\max } \leq 0.3 \%\right)$, отсюда можно определить максимальное отклонение оптической толщины слоя в процессе изготовления. Из рис. 3 видно, что чем быстрее величина пропускания меняется вблизи точки экстремума, тем меньше отклонение оптической толщины слоя. В работе [9] рассмотрен метод использования нескольких контрольных длин волн для уменьшения отклонения оптических толщин слоев в процессе их изготовления. В настоящей работе описан метод выбора показателя преломления стекла, из которого изготавливается подложка, для контроля слоя, изготовленного из материала с известным показателем преломления.

Из рис. 4 видно, что чем сильнее различаются показатели преломления материалов, из которых изготовлены подложка и слой, тем быстрее величина пропускания меняется вблизи точки экстремума, что позволяет формировать слои с минимальным отклонением от расчетного значения. Следовательно, для изготовления $i$-го слоя необходимо выбрать материал, который бы удовлетворял описанному выше требованию.

Для иллюстрации метода рассмотрим зеркальную (или фильтрующую) конструкцию в виде SHLHL...HL, где $\mathrm{S}-$ подложка, $\mathrm{H}$ и $\mathrm{L}-$ слои, изготовленные из материалов с высоким $n_{\mathrm{H}}$ и низким $n_{\mathrm{L}}$ показателями преломления [1-6]. В этом случае выбираются подложки $\mathrm{S}_{1}$ и $\mathrm{S}_{2}$, изготовленные из стекла с низким и высоким показателями преломления, такими, что $1.38 \leq n \leq 2.3$, а разница между величинами $n_{\mathrm{H}}-n_{\mathrm{S}_{1}}$ и $n_{\mathrm{S}_{2}}-n_{\mathrm{L}}$ должна быть максимальной. При изготовлении слоя с высоким
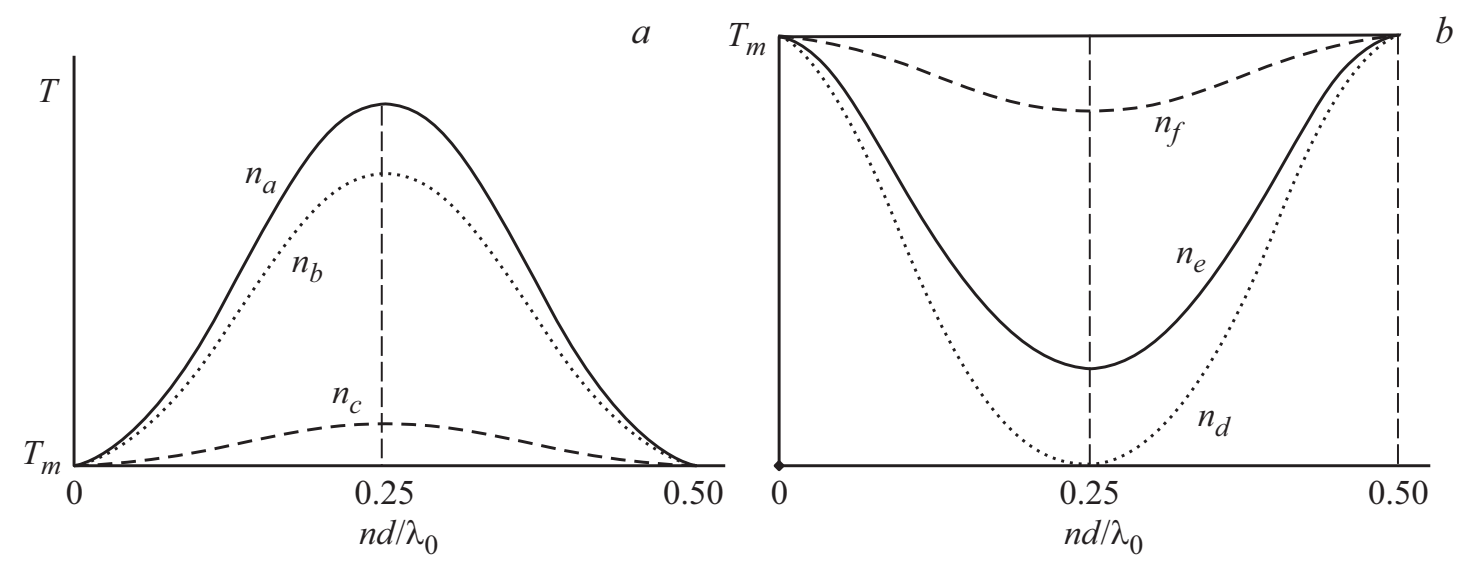

Рис. 4. Зависимости характеристик энергетического коэффициента пропускания слоя от его оптической толщины, выраженной в долях длины волны, при разных показателях преломления: $(a) n_{a}<n_{b}<n_{c}<n_{S}$, (b) $n_{d}>n_{e}>n_{f}>n_{S}$. 
показателем преломления для контроля оптической толщины слоя используем подложки $\mathrm{S}_{1}$. После завершения формирования слоя с высоким показателем преломления подложка, по которой производился контроль, убирается из зоны потока пленкообразующего материала, a в эту зону вводится вторая подложка $\mathrm{S}_{2}$, по которой производится контроль формирования слоя с низким показателем преломления.

\section{Заключение}

В работе представлены метод увеличения устойчивости спектральных характеристик интерференционных покрытий к отклонениям в параметрах слоев, входящих в их состав слоев, и метод использования нескольких образцов при контроле формирования слоев, входящих в состав интерференционного покрытия для уменьшения отклонения оптических толщин в процессе изготовления интерференционных покрытий.

\section{Список литературы}

[1] Губанова Л.А., Путилин Э.С. Оптические покрытия. Учебник для вузов. СПб.: Лань, 2016. 286 с.

[2] Macleod H.A. Thin-film optical filter. N.Y.: McGraw, 2010. $772 \mathrm{p}$.

[3] Dombrowski J.A. Optical properties of films and coating. V. 1. NY:: McGraw, 1995. P. 42.

[4] Крылова Т.Н. Интерференционные покрытия. Л.: Машиностроение, 1973. 224 с.

[5] Кокс Джс.Т., Хасс Г. // Физика тонких пленок.1967. Т. 5. М.: Мир, С. 46-83.

[6] Яковлев П.П., Мешков Б.Б. Проектирование интерференционных покрытий. М.: Машиностроение, 1987. 192 с.

[7] Sullivan B.T., Dobrowolski J.A. //Appl. Opt. 1992. V. 31. N 31. C. $3821-3835$.

[8] Schulz U., Schallenberg U.B., Kaiser N. // Appl. Opt. 2003. Vol. 42. N 7. C. $1346-1351$.

[9] Cheng-Chung Lee., Kai Wu. // Opt. Expr. 2005. V. 13. N 13. C. $4854-4861$. 